\title{
Gene disruption of the calcium channel Orai1 results in inhibition of osteoclast and osteoblast differentiation and impairs skeletal development
}

\author{
Lisa J Robinson ${ }^{1,5}$, Salvatore Mancarella ${ }^{2,5}$, Duangrat Songsawad ${ }^{1}$, Irina L Tourkova ${ }^{1}$, John B Barnett ${ }^{3}$, \\ Donald L Gill ${ }^{2}$, Jonathan Soboloff ${ }^{2}$ and Harry C Blair ${ }^{1,4}$
}

Calcium signaling plays a central role in the regulation of bone cells, although uncertainty remains with regard to the channels involved. In previous studies, we determined that the calcium channel Orai1 was required for the formation of multinucleated osteoclasts in vitro. To define the skeletal functions of calcium release-activated calcium currents, we compared the mice with targeted deletion of the calcium channel Orai1 to wild-type littermate controls, and examined differentiation and function of osteoblast and osteoclast precursors in vitro with and without Orai1 inhibition. Consistent with in vitro findings, Orai $1^{-1-}$ mice lacked multinucleated osteoclasts. Yet, they did not develop osteopetrosis. Mononuclear cells expressing osteoclast products were found in Orai $1^{-1-}$ mice, and in vitro studies showed significantly reduced, but not absent, mineral resorption by the mononuclear osteoclast-like cells that form in culture from peripheral blood monocytic cells when Orai 1 is inhibited. More prominent in Orai $1^{-/}$mice was a decrease in bone with retention of fetal cartilage. Micro-computed tomography showed reduced cortical ossification and thinned trabeculae in Orai1 ${ }^{-1-}$ animals compared with controls; bone deposition was markedly decreased in the knockout mice. This suggested a previously unrecognized role for Orai 1 within osteoblasts. Analysis of osteoblasts and precursors in Orai $1^{-l-}$ and control mice showed a significant decrease in alkaline phosphatase-expressing osteoblasts. In vitro studies confirmed that inhibiting Orai1 activity impaired differentiation and function of human osteoblasts, supporting a critical function for Orai1 in osteoblasts, in addition to its role as a regulator of osteoclast formation.

Laboratory Investigation (2012) 92, 1071-1083; doi:10.1038/labinvest.2012.72; published online 30 April 2012

KEYWORDS: osteoblast; osteoclast; osteogenesis; osteopetrosis; STIM1

Calcium signaling plays a central role in the regulation of osteoclast differentiation, but the mediators of calcium signals in bone cell development remain to be fully defined. In monocytic osteoclast precursors, calcium signals activate calmodulin-dependent kinases and phosphatases leading to the activation of transcriptional regulators, including the cAMP response element-binding protein (CREB) and nuclear factor of activated $\mathrm{T}$ cells 2 (NFAT2, also denoted NFATc1). ${ }^{1,2}$ Calcium channels from several families, including inositol-1,4,5-trisphosphate receptors (IP3R), ryanodine receptors and transient receptor potential channels, have been implicated in osteoclast formation and function. ${ }^{2-4}$
We showed recently that the cell membrane calcium channel Orail, which is activated by endoplasmic reticulum (ER) calcium depletion, is required to support osteoclast development in vitro. ${ }^{5}$ Specifically, when Orail activity or expression is inhibited, formation of multinucleated osteoclasts from RANKL-treated human or murine monocytes is inhibited. ${ }^{5}$ In contrast, little is known with regard to the role of store-operated calcium entry in bone formation or osteoblast differentiation, ${ }^{4}$ although calcium signals are also important regulators of osteoblasts. ${ }^{6}$

In osteoclast precursors, as in many cell types, release of intracellular calcium stores triggers a subsequent influx of

\footnotetext{
${ }^{1}$ Department of Pathology, University of Pittsburgh School of Medicine, Pittsburgh, PA, USA; ${ }^{2}$ Department of Biochemistry, Temple University School of Medicine, Philadelphia, PA, USA; ${ }^{3}$ Department of Microbiology, Immunology and Cell Biology, West Virginia University School of Medicine, Morgantown, WV, USA and ${ }^{4}$ Veteran's Affairs Medical Center, Pittsburgh, PA, USA

Correspondence: Professor LJ Robinson, Department of Pathology, University of Pittsburgh School of Medicine, Scaife S707A, 3550 Terrace Street, Pittsburgh, PA 15261, USA or Professor HC Blair, Veteran's Affairs Medical Center, Scaife S705, 3550 Terrace Street, Pittsburgh, PA 15261 USA.

E-mail: robinsonlj@msx.upmc.edu or hcblai@@imap.pitt.edu

${ }^{5}$ The first two authors contributed equally to this work. 
extracellular calcium that is necessary for sustained calcium signaling. ${ }^{1,2}$ Our previous work showed that depletion of intracellular calcium stores activates STIM1-Orail-mediated calcium influx. ${ }^{4}$ STIM1 is a transmembrane calcium-sensing protein in the ER that undergoes a conformational change when ER calcium is depleted, resulting in aggregation and interaction with Orail in 'puncta' in the plasmalemma, Orai channel opening and calcium influx. ${ }^{78}$ Coupling of STIM1 and Orail produce highly selective calcium currents. ${ }^{7,9}$ Consistent with our findings, Kim et al ${ }^{10}$ demonstrated recently that knockdown of STIM1 expression or inhibition of IP3Rs with xestospongin C eliminated calcium oscillations required for osteoclastic differentiation in bone marrow macrophages treated with RANKL.

A small number of patients with Orail gene defects have been reported. Two of these cases have been classified as Immune Dysfunction with T-cell Inactivation Due to Calcium Entry Defect 1 (On-line Mendelian Inheritance in Man, file 612782). The predominant phenotype is that of immunodeficiency, and most patients die by 1 year of age as a result of infection. ${ }^{11,12}$ Early mortality has limited assessment of the skeletal consequences of the Orai mutations, but the patients are reported to show developmental delay compatible with the mouse phenotype. In addition, some patients are reported to show amelogenesis imperfecta, and skeletal dysmorphism has also been reported. ${ }^{11}$ We hypothesized that the developmental delay observed in these patients might reflect impaired skeletal development in the absence of Orail, potentially linked to the in vitro defect we observed in osteoclastogenesis. ${ }^{5}$ If so, specific calcium channel inhibitors might be useful to prevent osteoclastic bone damage in clinical settings such as inflammatory arthritis.

We investigated the role of Orail in bone development using an Orail ${ }^{-1-}$ (knockout (KO)) mouse. We compared the skeletal features of the $\mathrm{KO}$ mice to litter-matched controls with normal Orail (wild-type (WT)). This model is useful, but poor survival of Orai1 $1^{-1-}$ animals is a limiting factor. Our results confirm the importance of Orail in osteoclast development: formation of multinucleated osteoclasts and resorption of mineralized tissue were impaired in $\mathrm{KO}$ mice in vivo. Yet, osteopetrosis did not occur, apparently reflecting the presence of small osteoclast-like cells that retain a limited capacity for resorption of mineralized tissue as well as an unexpected defect in bone formation, reproduced by Orail inhibition in vitro. These findings together imply that Orail is necessary for normal bone formation as well as normal osteoclast differentiation and is a critical regulator of the skeleton.

\section{MATERIALS AND METHODS}

\section{Animals and Genotyping}

Orail $^{-1-}$ mice were originally generated by Gwack et $a l^{13}$ as previously described in detail; briefly, the Orail gene was disrupted by homologous recombination using B6/3 embryonic stem cells derived from C57BL/6 mice (TaconicArtemis, Koln, Germany); chimeric mice were generated by blastocyst injection and founders bred to C57BL/6 mice (TaconicArtemis). ${ }^{13}$ In the C57BL/6 background, homozygous Orail deficiency resulted in neonatal death. We therefore generated Orail ${ }^{-1-}$ mice in a mixed genetic background: heterozygous Orai1 ${ }^{+/-}$C57BL/6 mice were intercrossed for 1-6 generations with mice of the outbred ICR strain (Taconic), after which ICR Orail ${ }^{+/-}$heterozygous mice were mated to generate Orail ${ }^{-1-}$ mice, which survive for $\sim 4$ weeks. All animals were maintained in pathogen-free barrier facilities and used in accordance with IACUCapproved protocols. Mice were genotyped by PCR of tail DNA with primers: forward $5^{\prime}$-GGGTGTGGCGTATGCAAATAA CCT-3', reverse (WT) 5'-ACTCGAGCCGGTCTCCC-3'; and reverse (KO) 5'-TCGTACCACCTTCTTGGGACTTGA-3'. Products were separated on $2 \%$ agarose to show the $200 \mathrm{bp}$ WT and $300 \mathrm{bp}$ targeted allele products. Because Orai ${ }^{-1-}$ animals die by $\sim 4$ weeks, mice were killed at 3 weeks.

\section{Skeletal Radiography and Whole Mounts}

Skeletons were fixed overnight in 5\% formaldehyde and stored until needed in $70 \%$ ethanol at $-20^{\circ} \mathrm{C}$. Whole animal radiography used a Faxitron MX20 (Lincolnshire, IL, USA); images were recorded on film. micro-computed tomography $(\mu \mathrm{CT})$ was performed on a Viva CT40 (Scanco, Bassersdorf, Switzerland) with reconstruction by instrument-specific software. The scan slice increment was $10 \mu \mathrm{m}$. Reconstructions used a density cutoff of $150 \mathrm{mg} / \mathrm{cm}^{3}$. After removal of skin and viscera, the cartilage was stained with alcian blue, $0.03 \%$ in ethanol and $20 \%$ acetic acid, overnight, followed by alizarin red $0.03 \%$ in $1 \% \mathrm{KOH}$, for 30 days at $4{ }^{\circ} \mathrm{C}$. Skeletons were then washed and clarified in $20 \%$ glycerol with $1 \%$ $\mathrm{KOH}$.

\section{Histology, Antibodies, and Microscopy}

Decalcified paraffin-embedded tissue was cut at $6 \mu \mathrm{m}$, deparaffinized and stained with hematoxylin and eosin or used for antibody labeling. For alkaline phosphatase, after citrateEDTA antigen retrieval and blocking in PBS and 2\% BSA, sections were incubated with rabbit monoclonal anti-alkaline phosphatase antibody (Epitomics, Burlingame, CA, USA) at 1:100, washed and incubated with Cy3-labeled goat antirabbit IgG (Jackson ImmunoResearch, West Grove, PA, USA) at 1:500. Frozen sections were cut with carbide blades and sections captured using the CryoJane transfer system (Leica Microsystems, Bannockburn, IL, USA). To evaluate bone formation, mice received intraperitoneal calcein, $1 \mathrm{mg} / \mathrm{kg}$, $48 \mathrm{~h}$ before being killed, and frozen sections were examined by fluorescence microscopy. Tartrate-resistant acid phosphatase (TRAP) activity was assayed in frozen sections fixed in citrate at pH 5.4 with $40 \%$ acetone. Acid phosphatase was then determined in $670 \mathrm{mM}$ tartrate at $\mathrm{pH} 5.6$ using naphthol AS-BI phosphate substrate and fast garnet GBC to visualize the product as a red precipitate (Sigma, St Louis, MO, USA). Calcified tissue in sections was labeled by $1 \% \mathrm{AgNO}_{3}$ under ultraviolet light (von Kossa stain) until 
contrast of mineralized (black) with non-mineralized tissue was adequate $(\sim 30 \mathrm{~min})$, followed by eosin counterstain. Western blots were performed as described. ${ }^{14}$ Anti-Orail was O8264 rabbit polyclonal (Sigma) produced using a peptide immunogen corresponding to amino acids 288-301 of human Orail. Imaging used a Nikon TE2000 inverted microscope with a 14-bit $2048 \times 2048$ pixel monochrome chargecoupled device and RGB filters for color reconstruction (Spot, Sterling Heights, MI, USA). Green fluorescence (calcein) used excitation $450-490 \mathrm{~nm}$, a $510 \mathrm{~nm}$ dichroic mirror, and a $500-570 \mathrm{~nm}$ emission filter. For red fluorescence, excitation was $530-560 \mathrm{~nm}$, a $575 \mathrm{~nm}$ dichroic mirror and a $580-650 \mathrm{~nm}$ emission filter.

\section{Cell Culture}

Human CD14-positive monocytic cells were isolated and differentiated by RANKL treatment. ${ }^{14}$ Orail activity was inhibited with $50 \mu \mathrm{M}$ 3,4-dichloropropionanilide (ChemServices, West Chester, PA, USA $)^{5}$ using equivalent solvent (ethanol) in controls. To assay resorption, osteoclasts were grown on slides coated with mineralized matrix (BioCoat; BD Biosciences, San Jose, CA, USA). After 12 days, cells were removed and the matrix stained using $\mathrm{AgNO}_{3}$ to differentiate resorbed areas from intact matrix. Area resorbed was determined by computer-aided analysis (Fovea Pro; Reindeer Graphics, Asheville, NC, USA). Human osteoprogenitor cells (CC-2538) were from Lonza (Walkersville, MD, USA) and cultured in growth medium: Dulbecco's modified Eagle's medium with $10 \%$ fetal bovine serum. For osteoblast differentiation and matrix mineralization, confluent cultures were changed to medium supplemented with $200 \mathrm{nM}$ hydrocortisone, $10 \mathrm{mM}$ glycerol-2-phosphate, $50 \mu \mathrm{g} / \mathrm{ml}$ sodium ascorbate, $2 \mathrm{mM} \mathrm{CaCl}_{2}$ and $10 \mathrm{nM} \mathrm{1,25-dihydroxyvitamin} \mathrm{D} \mathrm{(Sigma).}$ Media were replaced every 2-3 days. Alkaline phosphatase activity was assayed using naphthol AS-MX phosphate substrate in citrate buffer ( $\mathrm{pH} 8$ ) with fast blue RR to visualize the product as a blue precipitate (Sigma). To stain matrix mineral, cultures fixed in 5\% formaldehyde at $\mathrm{pH} 8$ were incubated in $2 \%$ alizarin red S (Sigma) for $20 \mathrm{~min}$ at room temperature.

\section{Quantitative PCR}

Total RNA was isolated using phenol/guanidine isothiocyanate (Trizol Invitrogen); RNA was quantified by $A_{260}$. Reverse transcription used $500 \mathrm{ng}$ of total RNA, random hexamer primers and MMLV reverse transcriptase (SuperScript III; Invitrogen). The $20 \mu \mathrm{l}$ reactions contained $20 \mathrm{mM}$ Tris, pH 8.3, $50 \mathrm{mM} \mathrm{KCl}, 5 \mathrm{mM} \mathrm{MgCl}_{2}, 10 \mathrm{mM}$ dithiothreitol, $0.5 \mathrm{mM}$ of each dNTP and RNase inhibitor (40 U, RNaseOUT; Invitrogen). Primer extension was performed at $42{ }^{\circ} \mathrm{C}$ for $1 \mathrm{~h}$. A measure of $1 \mu \mathrm{l}$ aliquots were used for PCR reactions in a prepared polymerase mixture (Brilliant III SYBR Green master mix; Agilent, Santa Clara, CA, USA) with $2.5 \mathrm{mM} \mathrm{MgCl} 2$ and $400 \mathrm{nM}$ oligonucleotide primers. After $10 \mathrm{~min}$ at $95^{\circ} \mathrm{C}, 15 \mathrm{~s} 95^{\circ} \mathrm{C}$ and $1 \mathrm{~min} 60^{\circ} \mathrm{C}$ cycles were performed on a MX3000P (Stratagene). Relative mRNA quantity was calculated by comparative cycle threshold, ${ }^{14}$ with glyceraldehyde-3-phosphate dehydrogenase (GAPDH) as the control. PCR product sizes were verified by agarose gel electrophoresis. Primers: GAPDH (GenBank: AF261085) forward $5^{\prime}$-GAGTCAACGGATTTGGTCGT- ${ }^{\prime}$, reverse 5'-TTGATTTTGGAGGGATCTCG-3'; RUNX2 (GenBank AF001450) forward 5'-CCTCGGAGAGGTACCAGATG-3', reverse 5'-TTCCCGAGGTCCATCTACTG-3'; collagen type I $\alpha 1$ (Col1A1, GenBank Z74615) forward 5'-AGGGCCAAG ACGAAGACATCCC-3', reverse 5'-TGTCGCAGACGCAGA TCCG-3'; alkaline phosphatase (ALPL, GenBank BC021289) forward 5'-CCTTGCTCAСТСАСТСАСТCC- $3^{\prime}$, reverse $5^{\prime}$-T TTTTTTTGCCGTTCCAAAC-3'; RANKL (TNFSF11, GenBank AF013171) forward 5'-ATCGTTGGATCACAGCAC AT-3', reverse 5'-AGACTCACTTTATGGGAACCAGA-3'; osteoprotegerin (TNFRSF11B, GenBank U94332) forward 5'-AACGCCAACACAGCTCACAAGAAC-3', reverse 5'-TGC TCGAAGGTGAGGTTAGCATGT- ${ }^{\prime}$; and ORAI calcium release-activated calcium modulator 1 (ORAI1, GenBank AK027372) forward 5'-AGGTGATGAGCCTCAACGAGCA-3', reverse 5'-AGTCGTGGTCAGCGTCCAGCT-3'.

\section{Statistics}

Numerical data are shown as mean \pm s.d. and represent comparisons of three independent determinations unless specified. Student's $t$-test was used for individual comparisons; analysis of variance was performed for comparisons of multiple samples (Table 1).

\section{RESULTS \\ Effects of Orai1 Deletion on Skeletal Modeling, Bone Density and Structure}

Orail $^{-1-}$ mice $^{13}$ were identified by PCR of genomic DNA (Figure 1a). Orai1 ${ }^{-1-}$ mice were consistently smaller and showed less postnatal growth than WT littermates. Heterozygotes showed no prominent abnormalities. For the WT and heterozygote groups, variation in weight and size within groups exceeded intergroup differences (data not shown); this heterogeneity likely reflects the outbreeding of carriers. Since our purpose was to determine the major effects of

Table 1 Histomorphometry parameters for three pairs of Orai $^{-I-}$ mice and WT littermates, measured at 3 weeks

\begin{tabular}{lccc}
\hline Parameter & Orai1 $^{-1-}(n=3)$ & WT $(n=3)$ & $P$ value $^{\mathrm{a}}$ \\
\hline Bone volume/total volume & $0.177 \pm 0.050$ & $0.283 \pm 0.050$ & 0.06 \\
(BV/TV) (dimensionless) & & & \\
Trabecular thickness (Tb.Th), $\infty \mathrm{m}$ & $0.050 \pm 0.005$ & $0.063 \pm 0.005$ & 0.03 \\
Trabecular number (Tb.N), mm ${ }^{-1}$ & $3.484 \pm 0.700$ & $4.460 \pm 0.450$ & 0.11 \\
Trabecular spacing (Tb.Sp), mm & $0.245 \pm 0.067$ & $0.162 \pm 0.028$ & 0.11
\end{tabular}

Values are mean \pm standard deviation.

${ }^{\mathrm{a}} P$-values are from Student's $t$-test. 
Orail deficiency, heterozygotes were not evaluated further. Mouse skeletons were examined at 3 weeks, near the limit of survival for Orail $^{-1-}$ mice ( $\sim 4$ weeks). The skeleton of the $\mathrm{KO}$ mice is proportionately smaller than the WT mice (Figure 1b). However, the modeling of the skeleton showed no gross defects: individual bones were scaled to the animal size. This suggested that Orail deficiency did not affect the modeling of the cartilaginous skeleton and that growth plate activity, while diminished, is properly regulated in the $\mathrm{KO}$ mice. Analysis of radiographs, with comparison of ribs and vertebrae from WT and KO mice (Figure 1c), suggested decreased bone density in the KO mice (Figure 1c). Tooth eruption and gross morphology in Orail ${ }^{-1-}$ mice were similar to that of WT, except that the teeth in $\mathrm{KO}$ mice appeared slightly smaller. However, microscopic sections revealed that enamel deposition in the $\mathrm{KO}$ mice was reduced and irregular (Figure 1d) in keeping with observations of amelogenesis imperfecta in humans with Orail defects. ${ }^{11}$
Quantitative effects of Orail deficiency on bone were determined by $\mu \mathrm{CT}$. Vertebrae were compared because they have the most uniform trabecular structure. Orai ${ }^{-1-}$ vertebrae were not only much smaller than WT mice, but also the mineralized bone showed discontinuities suggesting persistence of cartilage (Figure 2a). Although mice normally retain some cortical cartilage at birth, by 3 weeks the cortex should be fully ossified, as was evident in the control mice. In addition, vertebrae are normally filled with trabecular bone at 3 weeks, but in the Orail $1^{-1-}$ mice trabecular bone was reduced (Figure $2 \mathrm{a}$ ), although some regional variation was seen in all mice. Quantitative analysis of Orai ${ }^{-/-}$vertebrae showed a significant reduction in trabecular thickness $(P=0.03)$ with a trend toward decreased bone volume as a fraction of total volume $(P=0.06)$ relative to the WT mice. There was also a trend toward reduced trabecular number $(P=0.11)$ in Orai1 $^{-l-}$ mice. That statistical significance was not reached for some parameters reflects high intragroup a

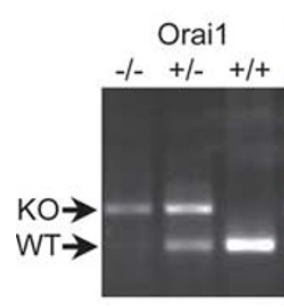

C
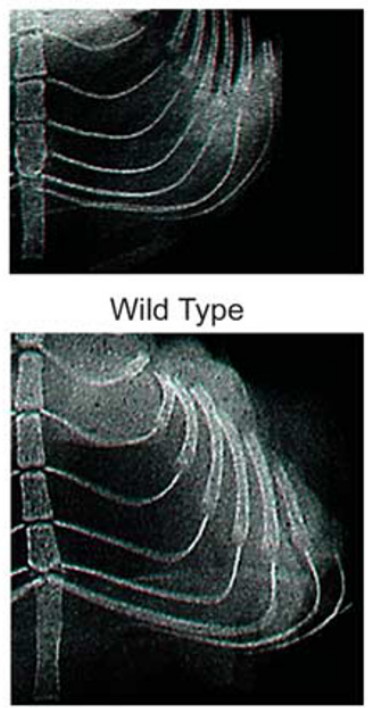

b

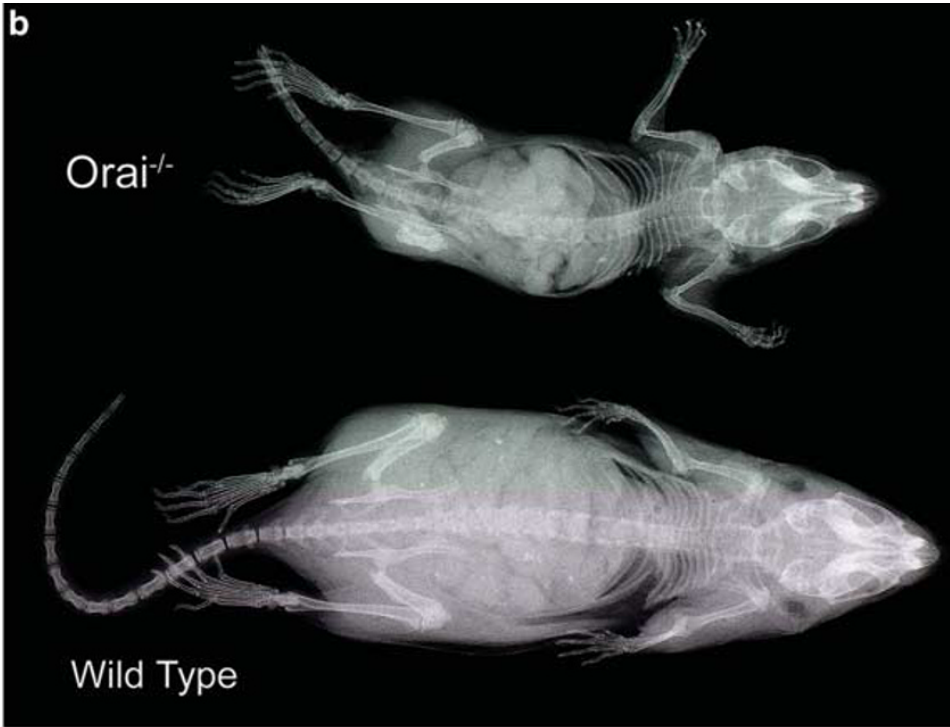

d
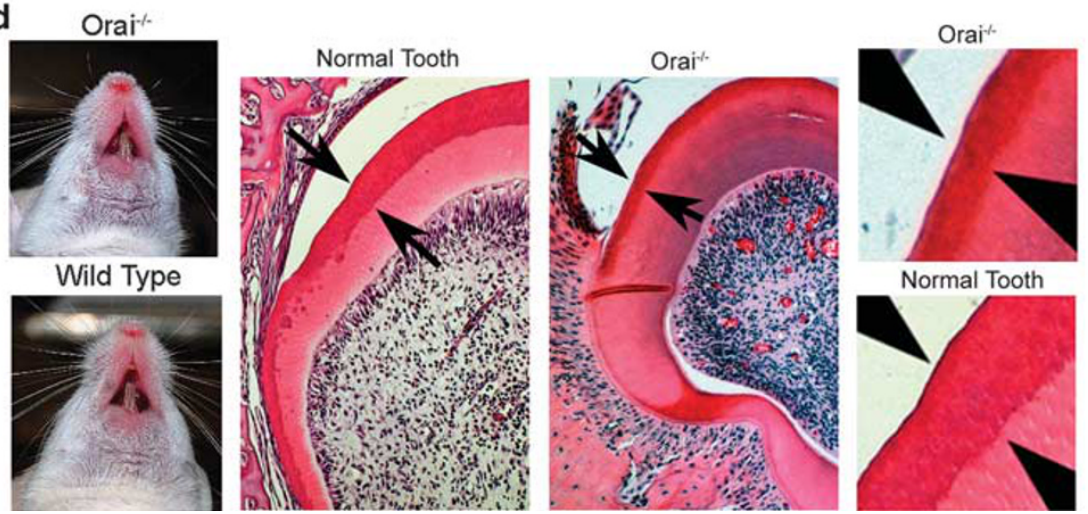

Figure 1 Skeletal phenotype and dentition of Orai $1^{-1-}$ mice. (a) Genotyping and gross appearance. The knockout (KO) mice were smaller than wild-type (WT) littermates. (b) Radiographs of Orai ${ }^{-1-}$ and WT mice showed no gross defects. Bones in the KO mice were decreased proportionately relative to overall mouse size. (c) Radiographs of thoracic vertebrae and ribs. There is reduced bone thickness in the Orai ${ }^{-/-}$animal. (d) Teeth in the Orai $1^{-/-}$mouse were slightly smaller than in WT mice, but sections showed thinning and irregularity of the enamel in Orai $1^{-1-}$. Similar sections through the gingival sulcus are shown to minimize the effect of plane of section. Left two frames, $450 \mu \mathrm{m}$; right frames, $110 \mu \mathrm{m}$ across. 
a
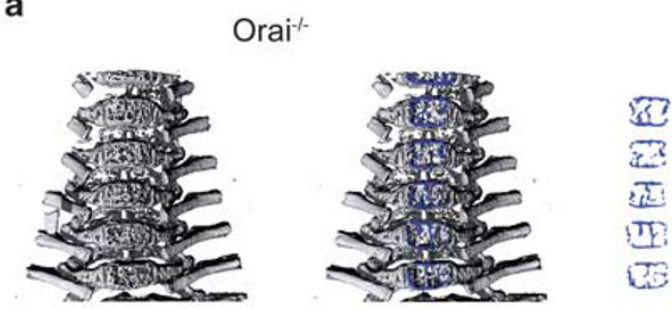

Wild Type
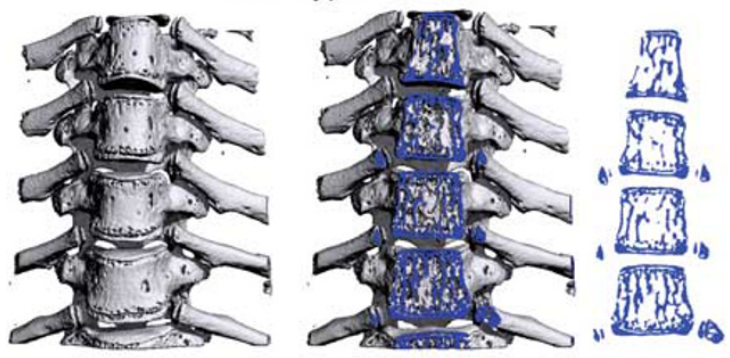

$\overline{1 \mathrm{~mm}}$
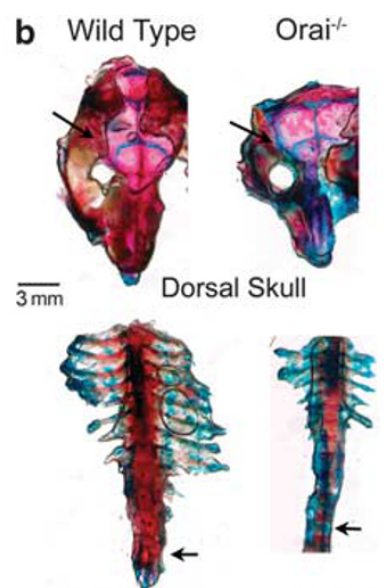

Thoracic and Lumbar Spine

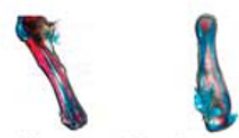

Ulna and Radius

Figure 2 Analysis of Orai $1^{-1-}$ skeletons by micro-computed tomography $(\mu \mathrm{CT})$ and alcian blue/alizarin red staining. (a) Thoracic vertebrae from wild-type (WT) and Orai $1^{-1-}$ mice by $\mu \mathrm{CT}$. Images (left and middle) are three-dimensional projections of the vertebrae (lowest is T12). Intact vertebrae (left), frontal sections with the virtual cut surfaces in blue (middle) and the cut surfaces separately (right). The vertebrae of Orai ${ }^{-1-}$ mice are smaller, show cortical discontinuities and reduced trabecular bone. (b) Skeletons stained with alcian blue to identify cartilage and alizarin red to stain mineral. Comparison of skulls (upper frames) revealed retained cartilage in the knockout (KO) mice (right) in areas mineralized in the WT mice (left). More cartilage remains in the skull around the nares in the Orai $1^{-1-}$, and cranial sutures are wider in the KO mice (arrows). The spines (middle frames) showed large regions of vertebrae remained cartilage in the KO mice (arrow) in contrast to WT mice, although there is regional variation. Replacement of cartilage by bone in the ulna and radius (lower frames) was incomplete in the KO mice. In controls, the shafts of the long bones were mineralized, with cartilage only at the joints.

variability and the low numbers of $\mathrm{KO}$ mice that survived long enough for analysis.

Cartilage and ossification in Orail ${ }^{-1-}$ and WT skeletons were then compared using alcian blue and alizarin red staining. Animals at 3 weeks could not be studied in whole mount, so key bones were dissected (Figure 2b). Alcian blue showed retained cartilage in bones of the skull in the Orail $^{-1-}$ mice. There was also increased cartilage and decreased bone in the vertebrae of the $\mathrm{KO}$ mice compared with controls, consistent with the $\mu \mathrm{CT}$ results, and the long bones of limbs were only partially mineralized in the Orail ${ }^{-1-}$ mice.

\section{Bone Formation and Degradation in KO and WT Animals}

Decreased bone and retained cartilage in the KO mice were confirmed in tissue sections (Figure 3a). Cartilage is normally present at the growth plates of the vertebral bodies (left and right sides of images; Figure 3a), but in the Orai ${ }^{-1-}$ mice cartilage persisted at surfaces of the vertebral body that, at 3 weeks, normally are replaced by bone. Within the vertebral bodies in the KO mice, trabecular bone was reduced, and trabeculae were thin. This apparent defect in bone formation was unexpected and complicated interpretation of resorptive activity. Evaluation of lamellar bone by polarized light showed a marked predominance of woven bone in the $\mathrm{KO}$ mice, in contrast to predominantly lamellar bone structure in WT mice (Figure 3b). Woven bone is characteristic of the initial bone formed, but normally it is promptly resorbed and replaced by lamellar bone. Remodeling to mature lamellar bone depends on osteoclastic resorption of the woven bone. Prominent woven bone is consistent with reduced bone resorption and impaired osteoclastogenesis. However, marrow space was present in the bones of the $\mathrm{KO}$ mice, in contrast to the osteopetrotic bone that results from complete absence of osteoclast function.

The predominance of woven bone in the Orail mice might be driven by reduced recruitment of osteoclasts, perhaps related to slow bone formation, or might indicate a primary osteoclast defect. Consistent with decreased bone resorption in the KO mice, TRAP activity, which was abundant at the bone surface in the WT mice, was markedly reduced in Orai1 $^{-1-}$ mice (Figure 3c). Few TRAP-positive cells were present and those that were present were small $(7-10 \mu \mathrm{m}$ in diameter) (Figure 3d, arrows). This suggested that some features of osteoclast differentiation, including TRAP expression, were preserved in the Orai1 ${ }^{-l-}$ mice, but fusion of pre-osteoclasts to form large multinucleated cells was markedly inhibited, consistent with results from our previous in vitro studies. ${ }^{5}$ The absence of adequate osteoclast function was also reflected in the presence of mineralized cartilage in the $\mathrm{KO}$ mice, as mineralized cartilage is normally rapidly degraded by osteoclasts and essentially absent from WT mice at 3 weeks (data not shown).

The lack of typical osteopetrosis, however, suggested some retention of osteoclastic function. Although efficient bone 

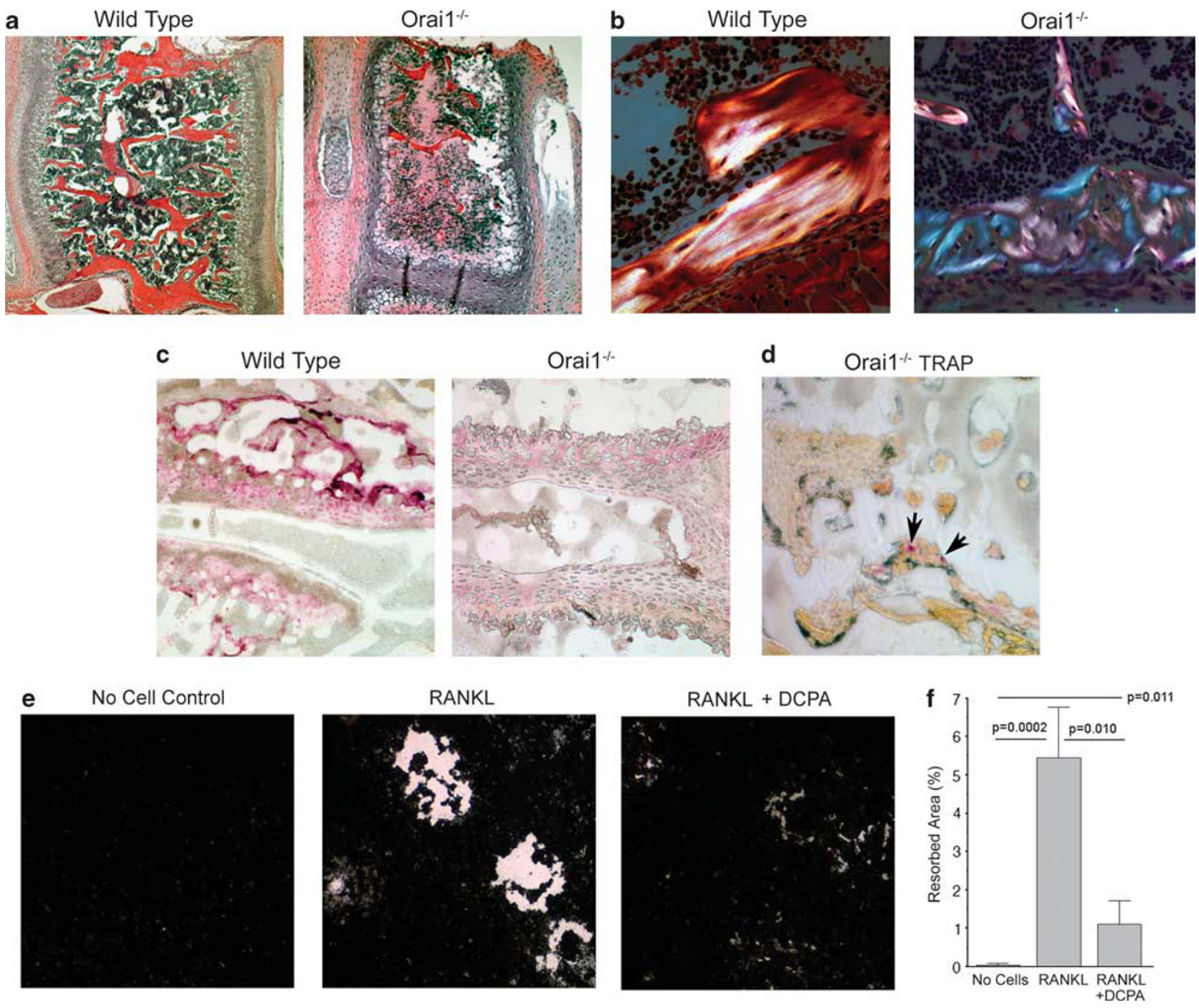

Figure 3 Bone, cartilage and osteoclasts in sections of wild-type (WT) and knockout (KO) bone, and osteoclast activity in vitro when Orai1 activity is suppressed. (a) Increased cartilage and decreased bone in KO mice. Decalcified sections from KO (right) and WT (left) third lumbar vertebrae stained with hematoxylin and eosin. In the Orai $1^{-1}$, the vertebral body retains cartilage on all sides, and has sparse, thin trabeculae. The control retains cartilage only at growth plates (gray, laterally) and has robust trabeculae. The growth plates are at the left and right sides of each frame. Images are $1.8 \mathrm{~mm}$ across. (b) Retention of woven bone in Orai ${ }^{-1-}$ mice. Collagen bundles are visualized by birefringence in polarized light. The cortex of a WT mouse vertebra (left) is mainly mature lamellar bone (parallel layers of collagen, bright). The KO mice retain woven bone (criss-cross pattern). Normally, the first bone synthesized when cartilage is replaced is woven bone, but woven bone is resorbed and replaced by lamellar bone. Images are $300 \mu \mathrm{m}$ across. (c) Decreased tartrateresistant acid phosphatase (TRAP, magenta) in cartilage growth plates of Orai ${ }^{-1-}$ mice. Histochemical staining was performed on frozen sections of vertebrae from WT (left) and KO (right) mice. The sections are centered on intervertebral discs and show the cartilaginous growth plates, which normally have high osteoclastic activity. Prominent TRAP is seen in WT, but much less in the KO mice. Images are $750 \mu$ m across. (d) TRAP-expressing cells in Orai ${ }^{-1-}$ mice. Higher power showed that the TRAP-positive cells (arrows) in Orai ${ }^{-1-}$ are single small cells, generally not multinucleated. Image width is $750 \mu \mathrm{m}$. (e) Reduced mineral resorption by osteoclasts differentiated in vitro when Orai1 is inhibited. Human osteoclast precursors were treated with RANKL to induce osteoclast differentiation, in the presence (right) or absence (middle) of the Orai1 inhibitor $\mathrm{N}$-(3,4-dichlorophenyl)-propanamide (DCPA), on slides coated with calcified matrix. Substrate resorption was evaluated by silver nitrate staining. Areas where substrate was resorbed are clear. Resorption was inhibited by the addition of $50 \mu \mathrm{M} \mathrm{DCPA}$ (right), compared with control (center). A no-cell control (left) has no resorption tracks. Note also that the resorption tracks in the Orai 1 inhibited cultures are thin and thread-like, consistent with resorption by small, mainly mononuclear, cells. This reflects the paucity of multinucleated osteoclasts when Orai1 is inhibited. (f) The area of degraded mineral substrate. Cleared matrix area in cultures without cells (left), RANKL-treated control cells (center) and cells treated with RANKL and the Orai1 inhibitor (right) are shown. DCPA resulted in a significant decrease $(P=0.01)$, in resorption. Activity in DCPA was clearly greater than the no-cell control $(P<0.01)$. In each case, resorption activity was measured in three cell cultures for each condition, and a second experiment showed equivalent results (not shown).

resorption requires multinucleated osteoclasts, it has been reported that less-differentiated mononuclear cells can resorb bone. $^{15,16}$ To test the hypothesis that the small TRAP-ex- pressing cells formed without Orail can degrade mineral, osteoclast precursors were cultured in RANKL to induce differentiation, without or with the Orail channel blocker 
$N$-(3,4-dichlorophenyl)-propanamide (DCPA). DCPA inhibits Orail activity by blocking its activation by Stim 1 in response to depletion of stored calcium. ${ }^{5}$ For these studies, cells were grown on slides coated with mineralized matrix. As we reported previously, TRAP-positive mononuclear cells but no multinucleated osteoclasts were found in DCPAtreated cultures. ${ }^{5}$ Nevertheless, trails of small resorption areas occurred in the DCPA-treated cultures, and Lysotracker staining (Invitrogen) confirmed production of acid by cells in the DCPA-treated cultures (not shown). Overall the total area resorbed was greatly decreased compared with control cultures without Orail inhibition (Figure $3 \mathrm{f}-\mathrm{g}$ ). These studies support the hypothesis that the TRAP-positive mononuclear cells seen in the $\mathrm{KO}$ mice have reduced but not absent capacity for degradation of mineralized matrix.

\section{Effects of Orai1 on Osteoblasts and Bone Formation}

The reduction in mineralized bone evident by $\mu \mathrm{CT}$ and apparent with alcian blue/alizarin red staining suggested that Orail deficiency might also impair the formation or function of osteoblasts. Indeed, it is possible that the relative prominence of woven bone in $\mathrm{KO}$ mice reflects impairment in bone formation as well as resorption. To evaluate the effects of Orail deficiency on bone formation in vivo, we used a single calcein injection to label mineral as it was deposited in the WT and KO mice. Dual calcein labeling was not practical because of the poor condition of the KO mice. WT mice showed strong calcein labeling on cortical and trabecular surfaces as expected, but labeling was markedly reduced in the Orail ${ }^{-I-}$ mice (Figure 4a). Where bone did form in the KO mice, calcein labeled the surfaces, as expected, but with thin labels consistent with a paucity of mineral deposition relative to WT bone. Decreased calcein labeling did not appear to reflect a specific defect in the mineralization of the bone: increased osteoid (non-mineralized bone) was not seen in the $\mathrm{KO}$ mice. To determine whether reduced bone formation might reflect decreased osteoblast numbers, osteoblasts were labeled in vertebral sections using antibody to alkaline phosphatase. The area labeled by alkaline phosphatase in the Orail $1^{-1-}$ mice was significantly decreased compared with results from controls (Figure 4b).

The effect of Orail deficiency on osteoblasts could be indirect, as osteoclasts and lymphocytes ${ }^{17,18}$ regulate osteoblasts and both appear compromised in the Orail $\mathrm{KO}$ mice. Alternatively, the bone formation defect in the Orail KO mice might indicate a novel role for Orail in the regulation of osteoblast differentiation. Orail expression has not previously been studied in osteoblastic cells. To investigate the possibility that Orail deficiency resulted in an intrinsic osteoblast developmental defect, we examined osteoblasts for Orail expression. To exclude the possibility that the deficiency was species specific, we tested human osteoprogenitor cells grown in the medium with or without ascorbate, glycerol-2-phosphate and hydrocortisone (factors that promote osteoblastic maturation and bone deposition). The presence of Orail protein in human osteoblasts was confirmed by western blotting (Figure 4c), which raised the possibility of an increase in Orail expression with osteoblastic maturation. Orail mRNA was detected by RT-PCR and measured relative to GAPDH message using quantitative PCR. We found Orai1 expression was present in cells from both culture conditions; again, there appeared to be relatively greater expression in the osteogenic medium (Figure 4d).

To evaluate the potential functional significance of Orail in osteoblasts, we examined the effects of the Orail inhibitor DCPA on human osteoblast differentiation in vitro (Figure 5a-d). As expected, in osteogenic medium, alkaline phosphatase activity was markedly increased, but this increase was significantly blunted by DCPA treatment (Figure $5 \mathrm{c}$ ), consistent with impairment of osteoblast maturation when Orai1 function is blocked. Mineralization was evaluated using the calcium-chelating dye alizarin red. After 3 weeks of culture in osteogenic medium without the inhibitor, mineralization was readily apparent; however, when Orail inhibitor was added, alizarin red staining revealed a marked reduction in mineralization (Figure 5d).

Osteoblastic differentiation was further evaluated using quantitative real-time PCR to compare expression of osteoblast markers. Results were evaluated by analysis of variance to identify statistically significant differences; $P$-values for comparison of cultures in differentiation medium without or with DCPA are shown in the figure with statistical results for additional comparisons reported in the figure legend. RNA was collected from cultures at the start of the treatment period (Ctl) and after 14 and 21 days, in growth or osteogenic differentiation medium, each with or without $50 \mu \mathrm{M}$ DCPA (Figure 6a-c). In growth medium, osteoblast expression of RUNX2, alkaline phosphatase and type I collagen was relatively low and showed little or no increase with time. In contrast, RUNX2 expression was markedly increased in cells cultured in differentiation medium, particularly at the earlier time point (Figure 6a); an increase in RUNX2 expression was still detected when DCPA was added to the differentiation medium, but was significantly decreased compared to cultures with differentiation medium plus vehicle control. Similarly, alkaline phosphatase expression was increased at 14 and 21 days in osteogenic medium without inhibitor, but was significantly lower in DCPA-treated cells (Figure 6b). The increase in expression of type 1 collagen in differentiation medium was also significantly inhibited by the addition of DCPA (Figure 6c). These results suggest an important role for Orail activity in osteoblast differentiation, and support the hypothesis that decreased bone in Orail KO mice may reflect an intrinsic osteoblast defect. This raised the further possibility that the osteoclast defects in Orail deficiency might reflect impaired osteoblast regulation of osteoclastogenesis. However, expression of RANKL and osteoprotegerin in osteoblast cultures was not significantly altered by Orail inhibition (Figure 6d). 
a

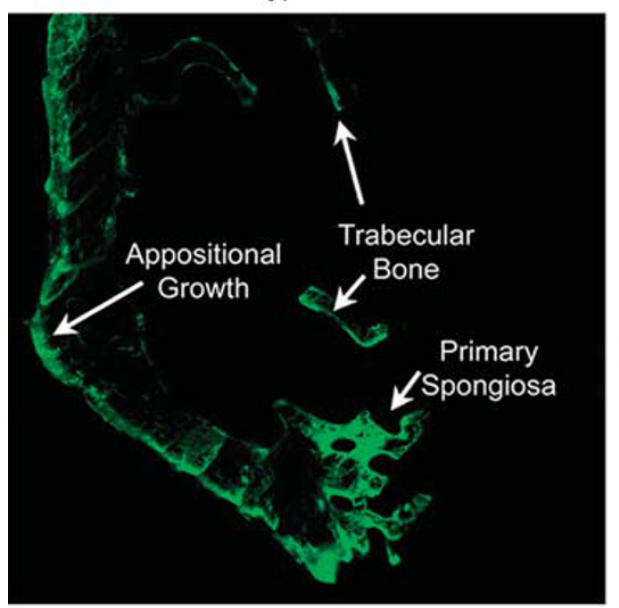

b

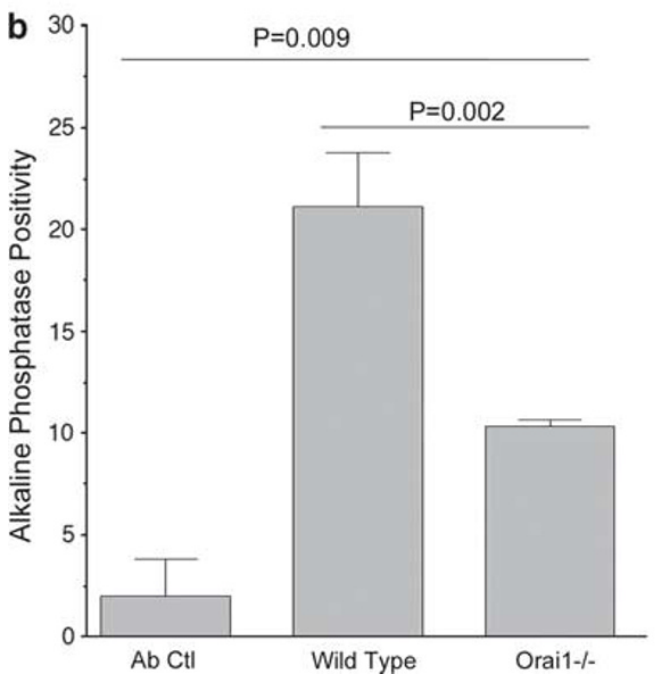

Orai1-/- Vertebra

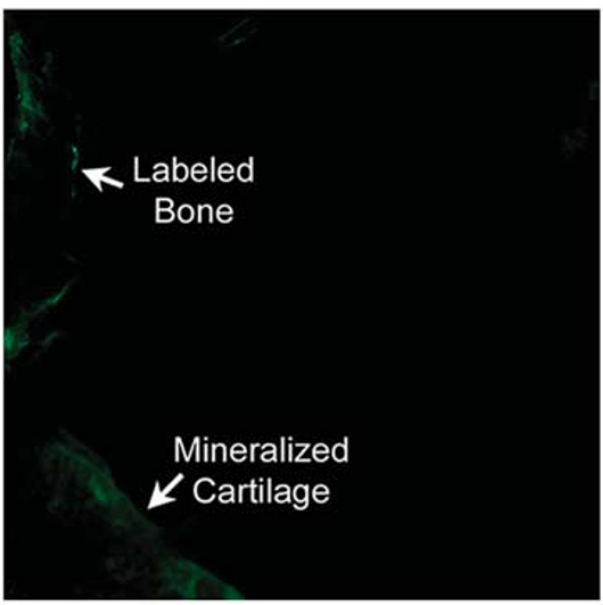

d

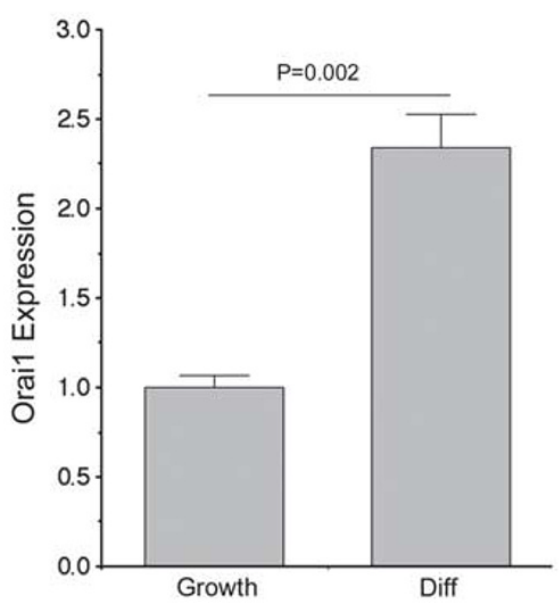

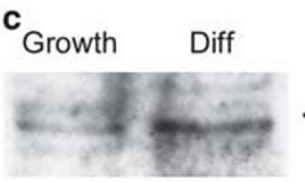

-Orai1 - Actin

Figure 4 Osteoblast activity in Orai ${ }^{-1-}$ mice. (a) Reduced mineralization in Orai ${ }^{-1-}$ mice. Fluorescent labeling of newly formed mineral in sections from wild-type (WT) (left) and knockout (KO) (right) mice 2 days after calcein injection. Sections are of vertebrae with growth plate at the bottom; each image is $250 \mu \mathrm{m}$ across. In the WT mice (left), there was calcein labeling along the bone surface (appositional growth), on trabeculae, and in the primary spongiosa, where bone replacing cartilage is remodeled. Labeled bone was markedly decreased in the Orai $1^{-1-}$ mice (right). Calcifying cartilage also labels with calcein, although these labels are diffuse. Some calcein labeling of mineralized cartilage is seen in the KO mice. Images are $250 \mu \mathrm{m}$ across. (b) Alkaline phosphatase in the vertebral bone of WT and Orai1-deficient mice. Decreased bone formation suggested that osteoblast numbers might be decreased. This was evaluated using an antibody for alkaline phosphatase that is highly expressed by active osteoblasts in bone. The total area labeling for alkaline phosphatase, measured in three sections of vertebrae, was decreased in Orai $1^{-1-}$ compared with the labeling measured in three sections from WT mice $(P=0.002)$.

(c) Osteoblast expression of Orai1 protein. Lysates from osteoblasts in basal (growth) or mineralizing (diff) medium were evaluated by western blotting with anti-Orai1, and blots re-probed with anti-actin to demonstrate equivalent sample loading. The results confirm expression of Orai1 by osteoblasts. Orai1 protein appeared increased in mineralizing osteoblasts in keeping with PCR results. (d) Osteoblast expression of Orai1 mRNA. Human osteoblasts in basal medium (Growth) or in differentiation medium containing ascorbate, glycerol-2-phosphate and hydrocortisone (Diff) were evaluated for Orai1 mRNA by quantitative $\mathrm{PCR}$, relative to glyceraldehyde-3-phosphate dehydrogenase (GAPDH), at 2 weeks. Orai1 message was present in the osteoblasts, with increased expression found in mineralizing cells $(n=5, P=0.002)$. Results at 3 weeks and in a repeat experiment were similar (not shown).

\section{DISCUSSION}

Our results identify Orai-mediated store-operated calcium influx, as a critical regulatory signal for both bone formation and degradation. Defects in Orail function were previously shown to cause T-cell abnormalities with immunodeficiency, ${ }^{19}$ mast cell abnormalities ${ }^{20}$ and altered platelet function. ${ }^{21}$ Decreased size was also consistently noted for the Orail-deficient mice, but the presence of underlying skeletal changes was not examined.
The Orail ${ }^{-l-}$ skeleton has several peculiar properties likely reflecting the combined effects of both osteoclast and osteoblast abnormalities. A defect in osteoclast maturation was expected ${ }^{5}$ and, indeed, mature multinucleated osteoclasts were essentially absent from the $\mathrm{KO}$ mice. Impaired osteoclast function was also suggested by the predominance of woven bone in the $\mathrm{KO}$ mice (Figure 3c), as osteoclast function is necessary for replacement of woven bone by lamellar bone. Yet, there appeared to be some calcified cartilage 
a

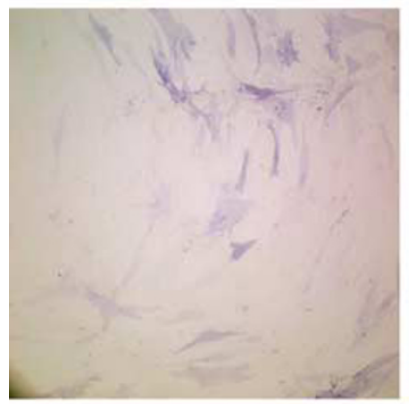

Growth

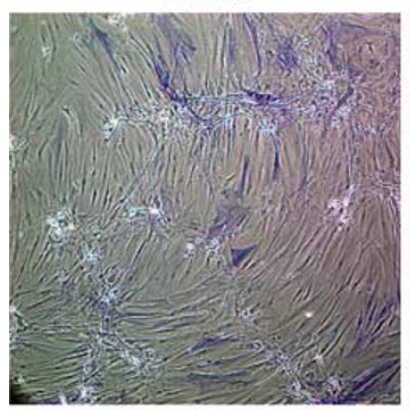

b

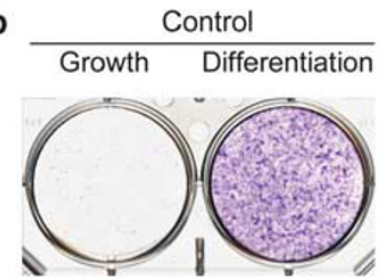

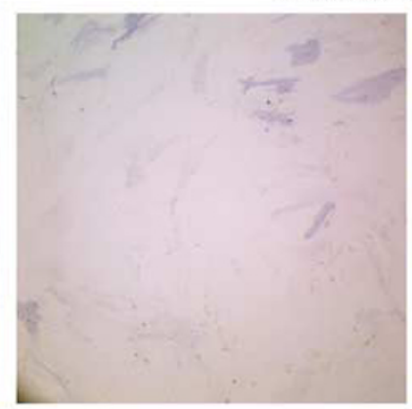

Growth + DCPA

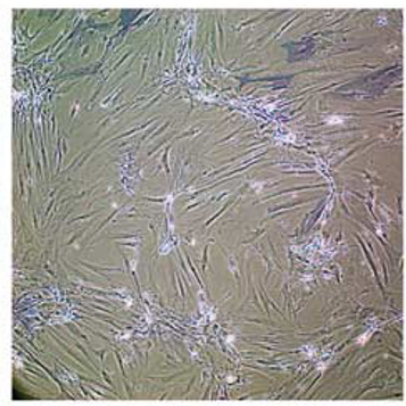

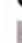

Growth Differentiation

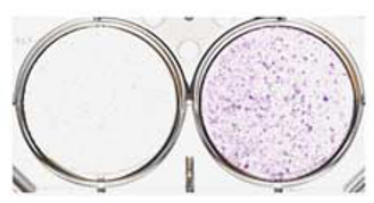

C

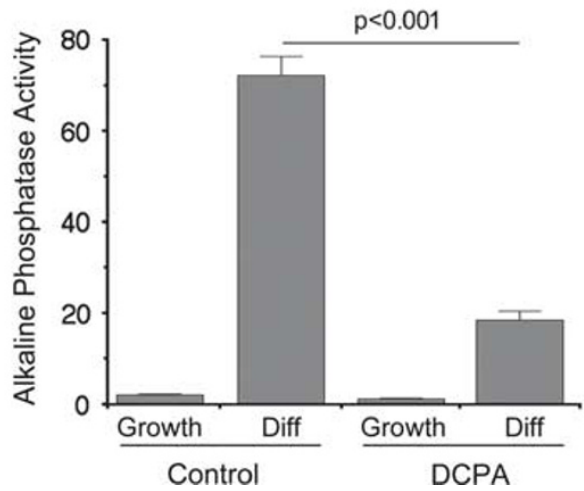

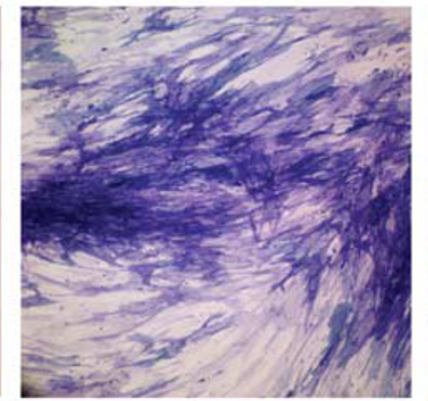

Diff

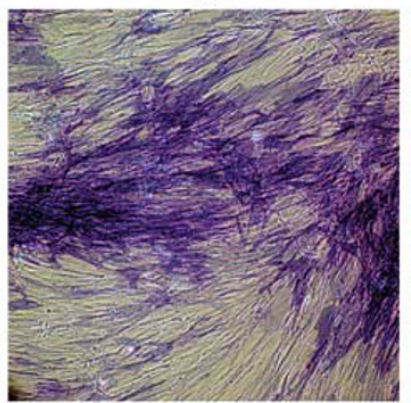

d
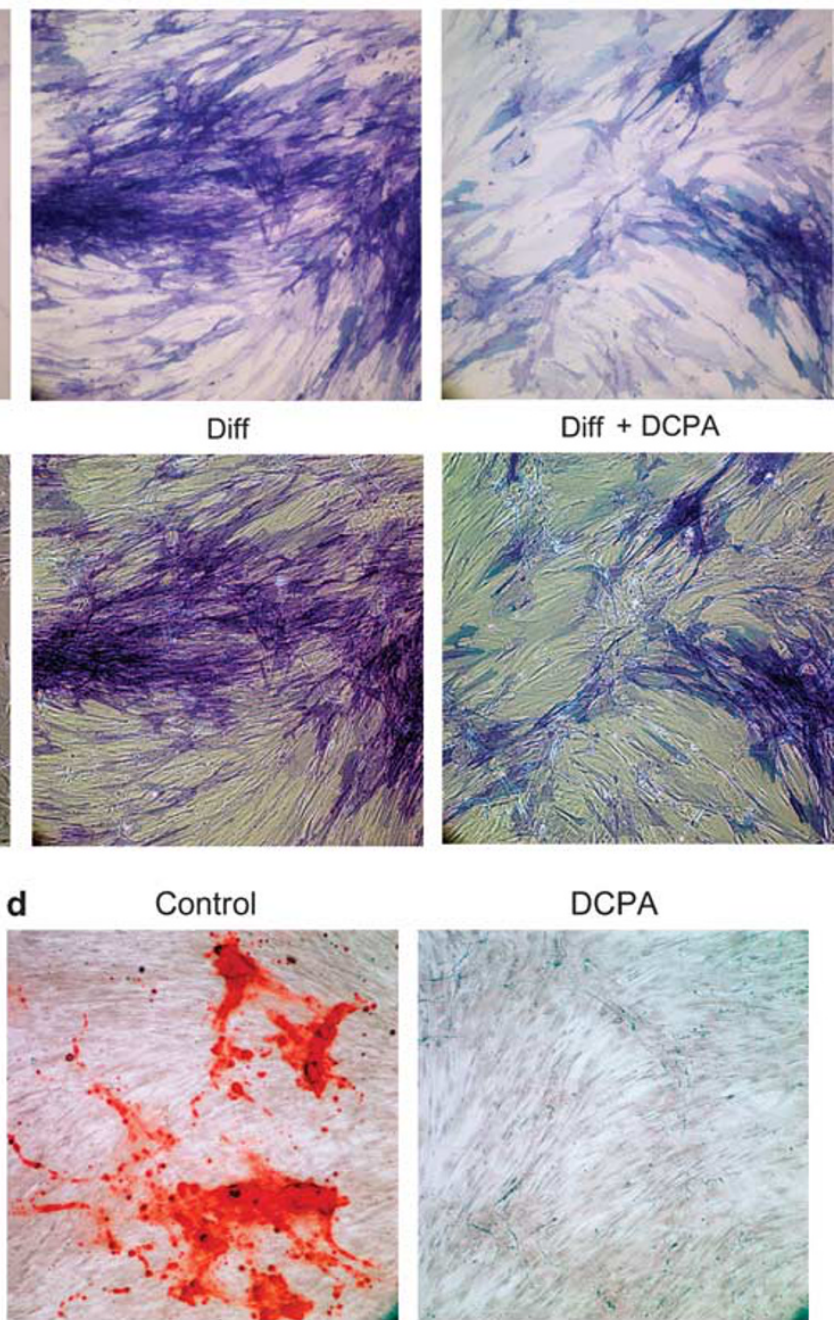

Diff + DCPA

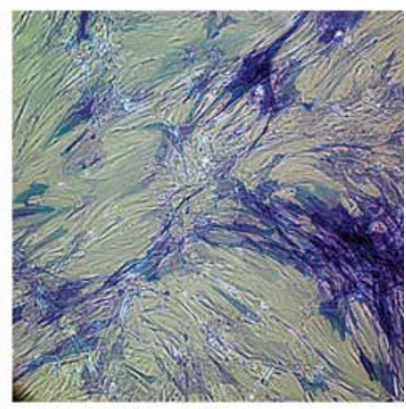

DCPA

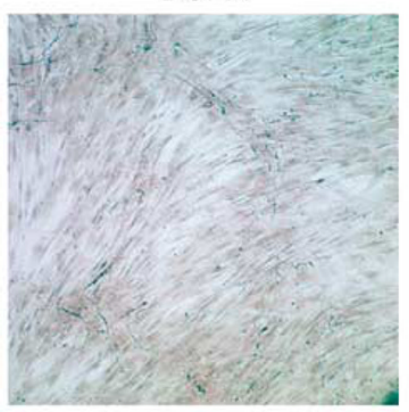

Alizarin Red

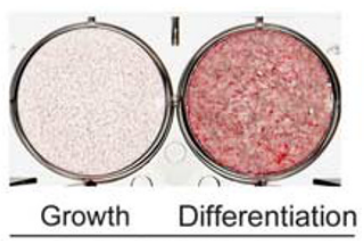

Control

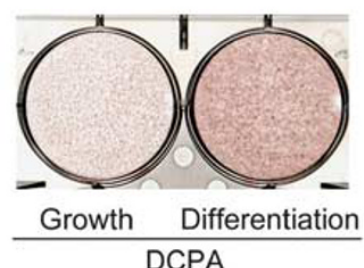

Figure 5 Effect of Orai1 inhibition on osteoblast differentiation and activity. (a) Effect of the Orai1 inhibitor $N$-(3,4-dichlorophenyl)-propanamide (DCPA) on alkaline phosphatase activity. Human osteoblasts were grown in basal medium (growth) or in medium with ascorbate, glycerol-2-phosphate and hydrocortisone (diff) to promote mineralization, with or without DCPA (or equivalent vehicle). Alkaline phosphatase activity (blue staining) was evaluated by light microscopy (upper row); a phase image of the same field is shown below. Little alkaline phosphatase activity was present in cells in growth medium. Alkaline phosphatase was substantially increased in cultures treated with ascorbate, 2-glycerol phosphate and hydrocortisone (diff), but this effect was blunted by DCPA. The phase images, showing cell monolayers with and without DCPA, indicate that the reduction in alkaline phosphatase did not simply reflect a difference in cell numbers in the presence of the inhibitor. Results were similar in repeat experiments (not shown). (b) Alkaline phosphatase in cultures of human osteoblasts. In situ enzyme activity is shown for cultures (left to right) in growth medium, growth medium plus DCPA, differentiation medium or differentiation medium plus DCPA. In differentiation medium, addition of DCPA reduced alkaline phosphatase activity. Wells are $3.5 \mathrm{~cm}$ across. (c) Alkaline phosphatase activity. For each condition in panel a, alkaline phosphatase is measured as signal at $450-490 \mathrm{~nm}$ in assays of replicate cultures. DCPA significantly reduced alkaline phosphatase in mineralizing osteoblasts $(n=4, P<0.001)$. (d) Mineral deposition by human osteoblasts is blunted by DCPA. Cells were cultured 3 weeks in basal medium (growth), or in mineralizing conditions (differentiation), each without or with DCPA. Alizarin red was used to stain calcium (bright red); representative cultures for each condition are shown (lower panel). As expected, little mineral was produced by osteoblasts in growth medium, with or without DCPA, but the photomicrographs show strong matrix labeling by alizarin red in cultures grown in differentiation medium without DCPA (control, upper left). However, when DCPA was added, alizarin red staining was mainly nonspecific (light red, upper right), without the focal strongly mineralized nodules that occur in differentiation medium alone. Wells are $3.5 \mathrm{~cm}$ in diameter; micrographs are $220 \mu \mathrm{m}$ across. 

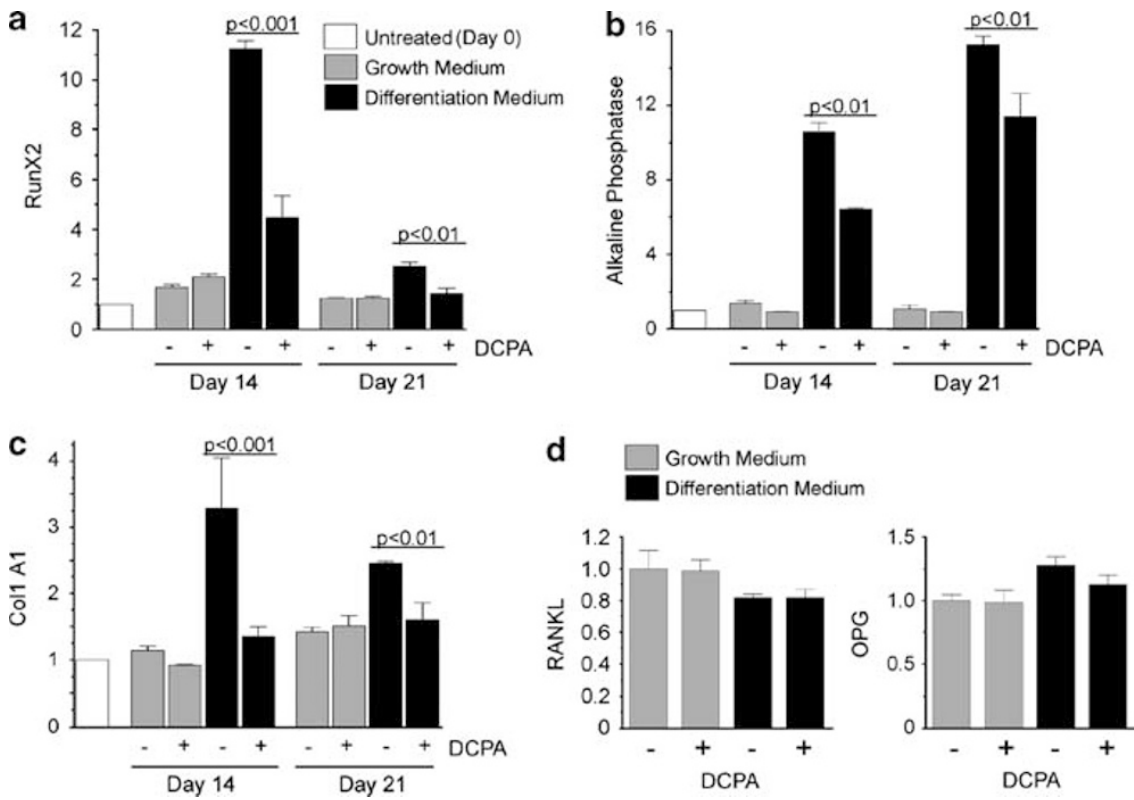

Figure 6 Effect of Orai1 inhibition osteoblast differentiation. Each assay shows expression relative to glyceraldehyde-3-phosphate dehydrogenase (GAPDH) mRNA in the same sample, mean \pm s.d., $n=3$. Differences were examined by analysis of variance. Repeat experiments gave similar results (not shown). (a) Expression of a transcriptional regulator of osteoblast differentiation, RUNX2. Human osteoblasts in growth medium, or in medium supplemented with ascorbate, glycerol-2-phosphate and hydrocortisone (differentiation medium) are compared, without or with the Orai1 inhibitor $\mathrm{N}$-(3,4-dichlorophenyl)propanamide (DCPA) $(50 \mu \mathrm{M})$. Cells in mineralizing medium for 2 weeks showed significant upregulation of RUNX2 compared with cultures in growth medium $(P<0.001$ without DCPA, $P<0.01$ with DCPA). The increase in RUNX2 with mineralizing medium was reduced by DCPA $(P<0.001)$. Although RUNX2 expression appeared lower in mineralizing medium at 21 days compared to 14 days, osteoblast RUNX2 expression was still significantly elevated $(P<0.01)$ in the absence of DCPA. RUNX2 expression by osteoblasts in mineralizing medium plus DCPA for 21 days was not significantly different from pretreatment or growth medium control cells. (b) Alkaline phosphatase mRNA. Alkaline phosphatase was significantly increased after $14(P<0.001)$ or 21 days $(P<0.001)$ in mineralizing medium compared with growth controls. Alkaline phosphatase expression by osteoblasts in mineralizing medium plus DCPA was reduced relative to mineralizing medium alone $(P<0.01)$. (c) Expression of type 1 collagen (collagen type I $\alpha 1$ (Col1A1)). Expression was increased for osteoblasts in mineralizing medium $(P<0.001)$ at 14 days. This increase was strongly attenuated by DCPA $(P<0.001)$, although DCPA-treated cells in mineralizing medium expressed $30 \%$ more Col1A1 than cells in growth medium with DCPA $(P<0.05)$. At 21 days, a reduced but still significant increase in Col1A1 expression was apparent in mineralizing medium without DCPA $(P<0.01)$, but collagen expression in cultures with DCPA was no longer significantly elevated compared with cells in the growth medium. (d) Expression of osteoclast regulatory proteins. RANKL and osteoprotegerin mRNA was assayed in human osteoblasts in growth $(\mathrm{Gr})$ or mineralizing medium (Dif), with or without DCPA, for 14 days. Unlike markers of osteoblastic differentiation, expression of RANKL and osteoprotegerin did not vary significantly with DCPA.

removal in the absence of Orail, and TRAP expression, although much decreased, was not absent from Orai1 ${ }^{-1-}$ bone. We speculated that the mononuclear TRAP-positive cells in the Orai KO mice, which comprise almost all of the TRAP-expressing cells in Orai1 ${ }^{-1-}$ bone, are capable of removing mineralized cartilage, albeit inefficiently. We therefore tested whether the TRAP-positive mononuclear cells resulting from RANKL stimulation with concurrent Orai1 inhibition ${ }^{5}$ could resorb mineralized matrix. These studies revealed markedly reduced but not absent matrix degradation (Figure 3e-f). Mononuclear cells with limited capacity for bone resorption have been reported previously: for example, macrophages from arthroplasty sites, although mononuclear cells, show a limited capacity for bone resorption. ${ }^{22}$

However, bone formation was also profoundly impaired overall in Orai $1^{-I-}$ mice (Figure 4) and this likely contributes to the lack of a dense skeleton in the $\mathrm{KO}$ mice despite impaired bone resorption. The skeleton of the $\mathrm{KO}$ animal is not only much smaller than controls, but also shows reduced or delayed ossification of bone. Dental enamel is also affected (Figure 1d), a finding consistent with amelogenesis imperfecta, which was reported for humans with Orail defects. ${ }^{11}$ These findings suggest that there might be a general defect in mineral-depositing cells in the absence of Orail.

The Orail ${ }^{-1-}$ bone phenotype resembles in some respects those resulting from FGFR3 defects. FGFR3 deficiency causes thanatophoric dysplasia with greatly reduced growth of long bones. However, cartilaginous defects, which were not identified in the Orail ${ }^{-l-}$ animal, are prominent with FGFR3 deficiency. ${ }^{23}$ To define fully the cellular defects underlying the skeletal changes in Orail deficiency, further detailed in vitro studies will be needed.

A major signaling pathway stimulating osteoclast differentiation proceeds from the TNF family receptor RANK through Traf6 to NF- $\kappa$ B activation, ${ }^{24}$ but calcium signals also appear to be critical. ${ }^{5,25-27}$ Recent work points toward primary regulation of ER calcium release by IP3R family channels in osteoclast precursors. ${ }^{10,24,28}$ A second phase of 
calcium signaling, also critical to differentiation, ${ }^{5,29}$ appears to be mediated by plasma cell membrane calcium channels: our results suggest that Orail is such a channel.

The working model for calcium signaling in developing osteoclasts involves activation of phospholipase $\mathrm{C} \gamma$ by RANKL and costimulatory signals, with a resulting rise in IP3-stimulating release of stored calcium. When $\mathrm{Ca}^{2+}$ is reduced in the endoplasmic reticulum, STIM1 undergoes a conformational change that causes it to aggregate with Orail in complexes at the plasma membrane called puncta, where Orail calcium channel activity is induced. ${ }^{7,9,30}$ The mechanism is known in some detail: a segment of STIM1 within the ER includes a $\mathrm{Ca}^{2+}$ binding $\mathrm{EF}$ hand domain with low $\mathrm{Ca}^{2+}$ affinity. ${ }^{31}$ Additional regulation is less well understood, but appears to involve intermediate signaling proteins such as CRACR2A. ${ }^{32}$ STIM1 mutations in humans have been related to immune defects, ${ }^{33}$ but, like defects in Orai $1,{ }^{19}$ have consequences in other tissues that are less well described. Interestingly, Masuyama et al ${ }^{34}$ identified a TRPV4 $\mathrm{Ca}^{2+}$ channel in osteoclasts and postulated a role for it in the terminal stages of osteoclast differentiation. Its function probably does not replace that of Orail, in the main (given the severe phenotype of the $\mathrm{Orai}^{-1-}$ animal). However, it might be responsible for the limited resorptive activity retained by the mononuclear TRAP-positive cells in Orail ${ }^{-1-}$ animals (Figure 3d).

In our previous in vitro studies, the most prominent osteoclast abnormality observed with Orail inhibition was a failure of precursor fusion; other components of osteoclast phenotype (eg expression of TRAP) appeared, at least partially, preserved. ${ }^{5}$ Consistent with in vitro findings, multinucleated osteoclasts were essentially absent from Orail KO mice, although some TRAP-positive mononuclear cells were detected. The mechanisms underlying impaired cell fusion due to Orail deficiency remain to be defined. In T cells, major targets of Orai-mediated calcium are the members of the NFAT family of transcriptional regulators, including NFATc1, that are activated by the calcium-dependent phosphatase, calcineurin. ${ }^{35}$ Osteoclastogenesis also involves NFATc1 activation through dephosphorylation by calcineurin, ${ }^{2,36}$ and the expression of genes such as TM7SF4 (which encodes the protein DC-STAMP), implicated in preosteoclast fusion, ${ }^{37}$ can be directly regulated by NFATc1. ${ }^{38}$ These observations suggest that the impaired osteoclast precursor fusion resulting from Orail deficiency might simply reflect inhibition of calcium-dependent NFATc1 activation.

Links between Orail, NFATc1 and osteoclast differentiation are also suggested by recent in vitro studies using a transformed murine cell line that can be induced by RANKL to form osteoclast-like cells. ${ }^{39}$ As in our studies of primary human osteoclast precursors, Orail inhibition in the cell line markedly reduced RANKL-stimulated cell fusion. Moreover, after Orail knockdown in the cell line, it was shown that NFATc1 activation, as detected by measurement of nuclear NFATc1 protein, was not significantly increased by RANKL treatment. However, the cell line studies showed preserved
RANKL induction of some NFATc1 target genes (eg TM7SF4), despite Orail inhibition. The cell line also showed significant RANKL induction of TRAP after Orail knockdown, although stimulation was less marked than in controls ${ }^{39}$ this result is consistent with the presence of residual TRAP-positive (albeit mononuclear) cells in the Orail KO animals (Figure 3).

It appears that the effects of Orail deficiency on osteoclastogenesis do not correlate exactly with the changes in the NFATcl activation even for genes known to be targets of NFATc1. Thus, while NFATcl activation is likely impaired by Orail deficiency in osteoclast precursors, as in $\mathrm{T}$ cells, mechanisms other than NFATc1 inhibition may contribute to the failure of Orail-deficient pre-osteoclast fusion. Normal osteoclastogenesis also requires calcium activation of calmodulin-dependent kinase (CamK) to stimulate phosphorylation of CREB; ${ }^{1}$ CamK may represent an additional target of Orail-mediated calcium signals in the osteoclast precursor. Further in vitro studies of Orail-negative cells will be needed to clarify mechanism(s).

Calcium is also an important osteoblast regulator, for example, through activation of calmodulin binding proteins such as CamK II. $^{6}$ NFAT proteins are also activated in osteoblasts by calcium-calcineurin-dependent dephosphorylation. Interestingly, increased extracellular calcium itself activates calcium signaling pathways through the calcium-sensing receptor. The calcium signaling pathways in osteoblasts appear to involve both release of calcium stores and influx of extracellular calcium, but the latter has been attributed to calcium entry through voltage-gated channels. ${ }^{6}$

We know of no precedents for calcium-release-activated calcium entry as a requirement for normal osteoblast differentiation, but the results of our in vitro studies suggest an intrinsic defect in osteoblast maturation when Orail calcium signaling is inhibited. It is also possible that osteoblastic effects of Orail deficiency are, at least in part, indirect. The defects in osteoclast formation may affect osteoblasts by interfering with osteoclast-derived regulatory signals. However, most mutations that inhibit osteoclast formation or function have not shown reduced bone formation. ${ }^{40}$ In addition, there are precedents for immune cells, chiefly lymphocytes, regulating skeletal activity, so the developmental delay in bone formation might in part reflect impaired immune regulation of mesenchymal stem cells or osteoblast maturation. ${ }^{41,42}$ As yet, these pathways are poorly defined and this should be regarded as speculative, particularly since the severe-combined immunodeficiency mouse has no changes in fetal skeletal development, although mild osteopenia develops as the animals age. ${ }^{43,44}$ The immune hypothesis is nonetheless attractive as it would tie the known immune defects of the Orai1 ${ }^{-1-}$ animal to the observed reduced osteoblast activity. The concept of immune regulation of bone cell differentiation has a strong following and is proposed as a new discipline, osteoimmunology. ${ }^{17,18}$ If we are successful in producing Orai1 ${ }^{-1-}$ MSC, the point should be 
easy to settle, although the mild skeletal phenotype of mice with severe-combined immunodeficiency implies that lymphocyte impairment cannot be the sole cause of defective bone formation in the Orai ${ }^{-1-}$ mouse.

In conclusion, in Orail ${ }^{-1-}$ animals, multinucleated osteoclasts were greatly decreased, although mononuclear cells expressing TRAP were present. Calcified cartilage was degraded but less rapidly, and bone turnover was absent or greatly reduced. There was also a profound reduction in synthesis of bone in Orail $^{-/-}$animals: cortical bone appeared thinned and trabeculae were reduced. The observed retention of fetal cartilage potentially reflects both impaired removal of mineralized cartilage and decreased or delayed bone formation. Overall, the results from the Orail $1^{-1-}$ mice indicate an essential role for the Orail calcium channel in differentiation and function of osteoclasts and osteoblasts in vivo.

\section{ACKNOWLEDGEMENTS}

This work was supported in part by the National Institutes of Health (USA) GM097335, ES011311, AG12951 and AR053566, and by the Department of Veteran's Affairs (USA).

\section{DISCLOSURE/CONFLICT OF INTEREST}

The authors declare no conflict of interest.

1. Sato K, Suematsu A, Nakashima T, et al. Regulation of osteoclast differentiation and function by the CaMK-CREB pathway. Nat Med 2006;12:1410-1416.

2. Negishi-Koga T, Takayanagi H. Ca ${ }^{2+}-\mathrm{NFATC1}$ signaling is an essential axis of osteoclast differentiation. Immunol Rev 2009;231:251-256.

3. Yaroslavskiy BB, Turkova I, Wang $\mathrm{Y}$, et al. Functional osteoclast attachment requires inositol-1,4,5-trisphosphate receptor-associated cGMP-dependent kinase substrate. Lab Invest 2010;90:1533-1542.

4. Blair $\mathrm{HC}$, Robinson $\mathrm{U}$, Huang $\mathrm{CL}$, et al. Calcium and bone disease. Biofactors 2011;37:159-167.

5. Zhou $Y$, Lewis $\mathrm{T}$, Blair $\mathrm{HC}$, et al. The role of calcium release activated calcium channels in osteoclast differentiation. J Cell Physiol 2011;226: 1082-1089.

6. Zayzafoon M. Calcium/calmodulin signaling controls osteoblast growth and differentiation. J Cell Biochem 2006;87:56-70.

7. Liou J, Kim ML, Do HW, et al. STIM is a $\mathrm{Ca}^{2+}$ sensor essential for $\mathrm{Ca}^{2+}$ store-depletion-triggered $\mathrm{Ca}^{2+}$ influx. Curr Biol 2005;15:1235-1241.

8. Soboloff J, Spassova MA, Tang XD, et al. Orai1 and STIM reconstitute store-operated calcium channel function. J Biol Chem 2006;281: 20661-20665.

9. Wu MM, Buchanan J, Luik RM, et al. $\mathrm{Ca}^{2+}$ store depletion causes STIM1 to accumulate in ER regions closely associated with the plasma membrane. J Cell Biol 2006;174:803-813.

10. Kim MS, Yang YM, Son A, et al. RANKL-mediated reactive oxygen species pathway that induces long lasting $\mathrm{Ca}^{2+}$ oscillations essential for osteoclastogenesis. J Biol Chem 2010;285:6913-6921.

11. McCarl CA, Picard C, Khalil S, et al. ORAI1 deficiency and lack of storeoperated $\mathrm{Ca}^{2+}$ entry cause immunodeficiency, myopathy, and ectodermal dysplasia. J Allergy Clin Immunol 2009;124:1311-1318.

12. Feske $S$, Gwack $Y$, Prakriya M. A mutation in Orai1 causes immune deficiency by abrogating CRAC channel function. Nature 2006;441: 179-185.

13. Gwack $Y$, Srikanth $S$, Oh-Hora $M$, et al. Hair loss and defective T- and B-cell function in mice lacking ORAl1. Mol Cell Biol 2008;28: 5209-5222.

14. Robinson LJ, Yaroslavskiy BB, Griswold RD, et al. Estrogen inhibits RANKL-stimulated osteoclastic differentiation of human monocytes through estrogen and RANKL-regulated interaction of estrogen receptor alpha with BCAR1 and Traf6. Exp Cell Res 2009;315: 1287-1301.

15. Itonaga I, Sabokbar A, Sun SG, et al. Transforming growth factorinduces osteoclast formation in the absence of RANKL. Bone 2004;34: 57-64.

16. Kahn AJ, Stewart CC, Teitelbaum SL. Contact-mediated bone resorption by human monocytes in vitro. Science 1978;199:988-990.

17. Takayanagi H. Osteoimmunology: shared mechanisms and crosstalk between the immune and bone systems. Nat Rev Immunol 2007;7:292-304.

18. Pacifici R. T cells: critical bone regulators in health and disease. Bone 2010:47:461-471.

19. Feske S, Picard C, Fischer A. Immunodeficiency due to mutations in Orai1 and Stim1. Clin Immunol 2010;135:169-182.

20. Vig M, DeHaven WI, Bird GS, et al. Defective mast cell effector functions in mice lacking the CRACM1 pore subunit of store-operated calcium release-activated calcium channels. Nat Immunol 2008;9: 89-96.

21. Braun A, Varga-Szabo D, Kleinschnitz C, et al. Orai1 (CRACM1) is the platelet SOC channel and essential for pathological thrombus formation. Blood 2009;113:2056-2063.

22. Chang JS, Quinn JM, Demaziere $A$, et al. Bone resorption by cells isolated from rheumatoid synovium. Ann Rhem Dis 1992;51: 1223-1229.

23. Ellman $\mathrm{MB}$, An HS, Muddasani $\mathrm{P}$, et al. Biological impact of the fibroblast growth factor family on articular cartilage and intervertebral disc homeostasis. Gene 2008;420:82-89.

24. Blair $H C$, Robinson $L$, Zaidi $M$. Osteoclast signalling pathways. Biochem Biophys Res Comm 2005;328:728-738.

25. Yaroslavskiy BB, Sharrow AC, Wells A, et al. Necessity of inositol $(1,4,5)$ trisphosphate receptor 1 and $\mu$-calpain in NO-induced osteoclast motility. J Cell Sci 2007;120:2884-2894.

26. Koga $T$, Inui $M$, Inoue $K$, et al. Costimulatory signals mediated by the ITAM motif cooperate with RANKL for bone homeostasis. Nature 2004;428:758-763.

27. Kim K, Kim JH, Lee J, et al. Nuclear factor of activated T cells $\mathrm{C} 1$ induces osteoclast-associated receptor gene expression during tumor necrosis factor-related activation-induced cytokine-mediated osteoclastogenesis. J Biol Chem 2005;280:35209-35216.

28. Kuroda $Y$, Hisatsune $C$, Nakamura $T$, et al. Osteoblasts induce $\mathrm{Ca}^{2+}$ oscillation-independent NFATC1 activation during osteoclastogenesis. Proc Natl Acad Sci USA 2008;105:8643-8648.

29. Robinson LJ, Blair HC, Barnett JB, et al. Regulation of bone turnover by calcium-regulated calcium channels. Ann NY Acad Sci 2010;1192: 351-357.

30. Deng X, Wang Y, Zhou Y, et al. STIM and Orai: dynamic intermembrane coupling to control cellular calcium signals. J Biol Chem 2009;284: 22501-22505.

31. Stathopoulos PB, Zheng L, Li GY, et al. Structural and mechanistic insights into STIM1-mediated initiation of store-operated calcium entry. Cell 2008;135:110-122.

32. Srikanth $\mathrm{S}$, Jung $\mathrm{H}-\mathrm{J}$, Kim K-D, et al. A novel EF-hand protein, CRACR2A, is a cytosolic $\mathrm{Ca}^{2+}$ sensor that stabilizes CRAC channels in T cells. Nat Cell Biol 2010;12:436-446.

33. Picard C, McCarl CA, Papolos A, et al. STIM1 mutation associated with a syndrome of immunodeficiency and autoimmunity. N Engl J Med 2009;360:1971-1980.

34. Masuyama R, Vriens J, Voets T, et al. TRPV4-mediated calcium influx regulates terminal differentiation of osteoclasts. Cell Metab 2008;8:257-265.

35. Oh-hora M. Calcium signaling in the development and function of T-lineage cells. Immunol Rev 2009;231:210-224.

36. Day CJ, Kim MS, Lopez CM, et al. NFAT expression in human osteoclasts. J Cell Biochem 2005;95:17-23.

37. Yagi M, Miyamoto T, Sawatini $Y$, et al. DC-STAMP is essential for cellcell fusion in osteoclasts and foreign body giant cells. J Exp Med 2005;202:345-351.

38. Kim K, Lee S-H, Kim JH, et al. NFATC1 induces osteoclast fusion via up regulation of ATP6V0d2 and the dendritic cell-specific transmembrane protein (DC-STAMP). Mol Endocrinol 2008;22:176-185.

39. Hwang $S-Y$, Putney JW. Orai1-mediated calcium entry plays a critical role in osteoclast differentiation and function by regulating activation of the transcription factor NFATc1. FASEB J 2012;26:1484-1492; 
available from: URL: www.fasebj.org/content/early/2011/12/21/fj.11 194399.full.pdf.

40. Lee $\mathrm{S}-\mathrm{H}$, Rho J, Jeong $\mathrm{D}$, et al. v-ATPase V0 subunit d2-deficient mice3 exhibit impaired osteoclast fusion and increased bone formation. Nat Med 2006;12:1403-1409.

41. Yamaza $T$, Miura $Y, B i ~ Y$, et al. Pharmacologic stem cell based intervention as a new approach to osteoporosis treatment in rodents. PLoS One 2008;3:e2615.
42. Terauchi M, Li JY, Bedi B, et al. T lymphocytes amplify the anabolic activity of parathyroid hormone through Wnt10b signaling. Cell Metab 2009;10:229-240.

43. Smetana K, Holub M. Ossification in nude mice: macroscopical study. APMIS 1990;98:729-734.

44. Smetana K, Holub M, Funda D. Ossification in nude mice: a histological, histochemical and immunohistochemical study. APMIS 1991;99: 1024-1030. 